\title{
Analysis of the application of biotechnological vaccines in immunology
}

\begin{abstract}
The survey provides information on the current status of immunization, vaccines used, and vaccine components.

The composition and general principles of the production of vaccine preparations are described. New generations of vaccines are characterized, indicating their disadvantages and advantages. The prospects of using new vaccines are analyzed. New generations of genetically engineered vaccines are absolutely safe due to the absence of residual pathogenicity, have a lower cost due to the reduction in the cost of industrial vaccine production, because there is no need for special equipment to protect personnel and the environment.
\end{abstract}

Volume 9 Issue | - 2021

\author{
Olga Y Makarova \\ Department of Molecular Biology and Biotechnology, VN \\ Karazin Kharkiv National University, Ukraine
}

Correspondence: Olga Y Makarova, Department of Molecular Biology and Biotechnology, VN Karazin Kharkiv National University, Kharkiv, Ukraine, Email makarovaolgae@gmail.com

Received: September 29, 2020 | Published: February 26, 202

Keywords: vaccines, antigens, immunization, immunologic drugs, genetic engineering, biotechnology

\section{Formulation of the problem}

As the protracted COVID-19 pandemic poses a huge global health challenge, the focus on personal hygiene and immunization is driving the search for new effective drugs to address the challenge. For several decades, infectious diseases have been a significant investment in national health authorities. According to the World Health Organization (WHO), vaccines are currently used to prevent about 30 infectious diseases. ${ }^{1-3}$ The arsenal of immunoprophylaxis includes more than 60 effective vaccine preparations, which have different compositions, methods of application and effectiveness. However, the latest techniques for the design of genetically engineered vaccines using virus-like particles, the creation of plant vaccines and new adjuvants (those substances that increase the immunogenicity of vaccines) are an important issue of modern immunology in order to improve prophylaxis measures. ${ }^{1,2,4,5}$ Licensed vaccines are thoroughly tested in several stages of testing, and only then are they become approved for use. In the pharmaceutical market, vaccines are regularly subject to repeated checks and controls. Scientists are constantly monitoring data to identify possible side effects after vaccination. ${ }^{1-3,6}$

\section{Research objects and methods}

Research on the development of vaccines by genetic engineering methods is carried out in many laboratories around the world. Significant progress has been achieved in the expression in bacteria and yeast of genes that encode the surface proteins of influenza and hepatitis B viruses, poliovirus, foot and mouth disease, rabies, etc. ${ }^{2,4,7}$ The high cost of producing new vaccines and the complexity of legal issues related to their use, and strict regulatory rules regarding the immunization of healthy people are significant barriers that affect pharmaceutical companies and deter them from entering the vaccine business. Therefore, in recent years, the number of vaccine manufacturers has decreased, which has led to a decrease in competition and a decrease in investment in this industry.

The current vaccines used in healthcare are the same as those developed many years ago, but they have been improved by advances in immunology to improve safety, tolerability and efficacy. Vaccines with improved characteristics have appeared, the production of which is impossible without improved technological processes. Some drugs developed decades ago (for example, the influenza vaccine) are still produced using outdated methods. ${ }^{2,4,7,8}$ The goal of modern immunology is the development and improvement of vaccines that are obtained using the latest technologies, in significant volumes, at the necessary speed that allows meeting the needs of mass vaccination activities. The revolutionary direction of modern vaccinology is the development of vaccines based on transgenic plants, in the genome of which the corresponding fragment of the genome of the pathogenic microorganism is embedded. It causes the formation of antibodies in the body, which form the immune system. Taking into account the use of these vaccine products in their raw form, the possibilities of growing vaccines on plants that do not require culinary processing before use are being investigated: tomatoes, bananas and lettuce. ${ }^{5,9-11}$

A vaccine is a drug that is administered to form active immunity against infectious diseases in the body of vaccinated people or animals. Vaccines are made from weakened or inactivated microorganisms, from the products of their vital activity or their antigens, obtained by genetically engineering or chemical means. In addition to vaccines from components of one type of microorganisms (monovaccines), in medical practice are used consisting of two or more components of microorganisms (polyvaccines). ${ }^{4}$ To prevent such dangerous infectious diseases as smallpox, diphtheria, tuberculosis, vaccines must be administered in accordance with the vaccination plan. In other cases, vaccination is carried out selectively, according to epidemic indications. For example, the population is vaccinated against plague, tularemia, Japanese and tick-borne encephalitis, brucellosis in natural foci.

Vaccines that are used for routine vaccinations are administered in a specific sequence. Newborns are vaccinated against tuberculosis (BCG), then they are vaccinated against diphtheria, tetanus and pertussis. Children are later vaccinated against smallpox, polio and measles. Some vaccines require revaccination, that is, readministration of the dose after a certain time. Thus, children are protected from the seven most dangerous bacterial and viral infections. ${ }^{4}$ Vaccines are based on projective antigens, parts of bacterial cells or viruses that ensure the development of a specific immune response. By their chemical nature these can be glycoproteins, proteins, lipopolysaccharide protein complexes. ${ }^{4}$ 
The vaccines also include sorbents, preservatives, fillers, stabilizers and nonspecific impurities: the substrate protein for the cultivation of viral vaccines, antibiotics in trace amounts and proteins of the serum of animals used in the cultivation of cell cultures. Vaccines adsorbed on aluminum derivatives (aluminum hydroxide or aluminum phosphate) are often used. Preservatives are added to all vaccines to ensure sterility when conditions for bacterial contamination arise (microcracks on ampoules or vials during transportation, storage of an open primary multi-dose vaccine package). An indication of the need for preservatives in vaccines is contained in the WHO recommendations. ${ }^{2,3,8,12}$ At the present stage of development of immunology as a fundamental medical and biological science, it became necessary to create fundamentally new approaches to the design of vaccines. Knowledge of the antigenic structure of the pathogen and the principles of the body's immune response to the pathogen or its components are used. ${ }^{2,7}$

Today, a new direction of development of artificially created antigenic determinants of microorganisms, biosynthetic vaccines obtained by genetic engineering methods is successfully developing. ${ }^{7}$ Chemical synthesis of peptides makes it possible to make affordable synthetic vaccine preparations that have advantages over traditional vaccines. They do not contain bacteria or viruses, their metabolic products, and cause an immune response with a narrow specificity. Complex technologies for growing viruses, their storage and the possibility of their replication in the body of those vaccinated with live vaccines are excluded, which, if virions are insufficiently inactivated, can lead to an outbreak of infection against which the vaccine is used. The introduction of synthetic vaccines devoid of carriers of genetic information (DNA and RNA molecules) eliminates this danger. ${ }^{12}$

The use of live vaccines limits their potential danger: there is always a certain risk of the return of virulence by the strains used. High antigenic variability of viruses (influenza virus) can lead to low efficacy of vaccines against virions of a particular strain. Influenza vaccines contain viral particles grown in chicken embryos. They have been extracted, inactivated and used as influenza vaccines for 70 years. In terms of antigenic composition, in accordance with WHO requirements, modern influenza vaccines are trivalent: they contain antigens of three influenza virus subtypes $\left(\mathrm{A} / \mathrm{H}_{1} \mathrm{~N}_{1}, \mathrm{~A} / \mathrm{H}_{3} \mathrm{~N}_{2}\right.$ and $\left.\mathrm{B}\right)$. The selection of the used virus strains is carried out in advance-several months before the start of the season of increased incidence. This makes the vaccine less likely to match the virus the vaccine is intended to prevent. The production of influenza vaccines is labor intensive, time-consuming, and requires the use of millions of eggs. Cultivation of the virus in chick embryos facilitates the random selection of strains that are able to grow under given conditions. One solution to this problem is to use cell cultures instead of embryos. This has a number of advantages: reducing the cost of vaccines, accelerating the process of obtaining a vaccine, increasing its controllability, producing significant volumes of products, and, importantly, eliminating cases of allergic reactions to the components of chicken eggs. . $^{1,6,8,13}$

It is generally impossible to obtain vaccine preparations from some viruses due to the unsatisfactory growth of the virion biomass. Therefore, it is promising to use synthetic peptides with antigenic determinants of their own strain and with antigenic activity, which can significantly increase the effectiveness of the vaccine and expand its valence. Thanks to the use of immunogenic proteins instead of whole cells of pathogens, the formation of immunity is ensured while reducing the reactogenicity of the vaccine and reducing its side effects. ${ }^{1,2,12}$ Genetic engineering approaches can be used to create molecular vaccines. The achievement of genetic engineering and biotechnology was the creation of recombinant antiviral vaccines containing hybrid nucleic acid molecules. They are obtained by embedding the genetic material of a microorganism into yeast cells that produce antigen. ${ }^{7,12}$

During the cultivation of yeast, the desired antigen is extracted from them, purified and a vaccine preparation is prepared (for example, this is how the "Euvax B» vaccine against hepatitis B is obtained). The introduction of such vaccines greatly simplifies the production process: viral DNA plasmids for vaccines are synthesized by $E$. coli bacteria during normal fermentation. This makes it possible to obtain highly purified stable preparations on a large industrial scale.

The production process of such vaccines is universal and does not depend on the nature of the pathogen: the difference between DNA vaccines is only the composition of the gene inserted into the bacterial cell. Therefore, recombinant antiviral vaccines are the newest generation of vaccines that have several advantages. They lack or significantly reduced the content of ballast components; they are absolutely safe due to the guaranteed absence of residual virulence; they have a lower cost, which is associated with a reduction in the cost of industrial production of vaccines, because there is no need for special equipment to protect personnel and the environment., ${ }^{2,412}$

Vaccines must meet the highest quality standards. Strict measures are applied to ensure appropriate quality and safety during the development, production, licensing, transportation, storage and use of vaccines, as well as when disposing of packages, ampules, syringes after vaccination activities. ${ }^{14}$ Like pharmacological drugs, vaccines are assessed for their effectiveness and potential harmful effects in vitro experiments and on laboratory animals. ${ }^{2,4}$ If there are doubts about the safety of a drug, the vaccine is not sent for licensing. About half of modern vaccine names have appeared in the last 25-30 years, which corresponds to the development of about one vaccine per year (for example, previously one vaccine was created in 5 years). The introduction of innovative techniques, improved technologies promises the success of vaccines currently being developed against HIV, malaria, hepatitis $\mathrm{C}$ and other serious diseases. ${ }^{2,12}$ The development and production of modern vaccines is carried out in accordance with high quality requirements based on WHO recommendations, which attracts the most authoritative experts from different countries to this issue.

\section{Conclusion}

An analysis of the rate of scientific and economic progress suggests that over the next decade, the rate of development of the vaccine business may exceed even the rate of development of the business of pharmaceuticals. According to forecasts of biotechnologists, for further work on new plant vaccines and their industrial production, in addition to funding, several more years of clinical trials are needed., ${ }^{910}$ In the future, recombinant preparations from genetically modified plants will replace bacterial and animal counterparts in the global pharmaceutical market. Plant vaccines will significantly improve the program of universal immunization of the population. .,9-11 $^{5}$

\section{Acknowledgments}

None.

\section{Conflicts of interest}

Authors declare that there is no conflict of interest. 


\section{References}

1. Onishchenko GG, Zverev VV, Katlinsky AV, et al. Tetravaccine-a new principled approach to preventing an influenza pandemic. Zh Mikrobiol Epidemiol Immunobiol. 2007;4:15-19.

2. Uchaikin VF, Shamsheva OV. Vaccine prophylaxis: Present and future. Geotar Med; 2001. 400 p.

3. Bronte BMV, Colombo MP, de Vinsentils A, et al. Antitumor vaccination: where we stand. Haematologica. 2002;85(11):1172-1206.

4. Ada G, Ramsay A. Vaccines, vaccination and the immune response. Medicine; 2002. 344 p.

5. Mason HS, Warzecha H, Tsafir MS, et al. Edible plant vaccines: applications for prophylactic and therapeutic molecular medicine. Trends $\mathrm{Mol}$ Med. 2002;8(7):324-329.

6. Govorkova EA, Webby RJ, Humberd J, et al. Immunization with reverse- genetic-produced $\mathrm{H} 5 \mathrm{~N} 1$ influenza vaccine protects ferrets against homologous and heterologous challenge. J Infect Dis. 2006;194(2):159167.

7. Maniatis T, Frig E, Sambrook J. Methods of genetic engineering: Molecular cloning. Mir; 1994. 480 p.
8. Bardiya N, Bae JH. Influenza vaccines: recent advances in production technologies. Appl Microbiol Biotechnol. 2005;67(3):299-305.

9. Daniell H, Streatfield S, Wycoff K. Medical molecular farming: production of antibodies, biopharmaceuticals and edible vaccines in plants. Trends in Plant Sci. 2001;6(5):219-226.

10. Mor TS, Moon YS, Palmer KE, et al. Geminivirus vectors for high level expression of foreign proteins in plant cells. Biotechnol Bioeng. 2003;81(4):430-437.

11. Yu J, Langridge WH. A plant-based multicomponent vaccine protects mice from enteric diseases. Nat Biotechnol. 2001;19(6):548-552.

12. Delvig AA, Semenov BF, Rosenquist E, et al. Neisseria Meningitidis: from antigenic structure to a new generation of vaccines. Medicine; 2000. 217 p.

13. Dorshna A, Naihin AN, Petukhova GD, et al. Systemic humoral and cellular immune response in experimental influenza infection and vaccination. Medical Immunology. 2006;8(1):3-4.

14. Kolyshkin VM, Baldin S Yu, Nochevny VT. Some aspects of transportation and storage of MIBP in the "Cold Chain" system. Epidemiology and Vaccine Prevention. 2006;5:59-63. 